\title{
Una visión de la crisis de la industria sedera toledana en el primer tercio del siglo XVII: el Memorial de Juan González de Vatres Sotomayor
}

\author{
Alfonso Cebrián Rey *
}

RESUMEN

La industria sedera toledana del siglo XVII es ejemplo de una actividad determinada, objeto de numerosos escritos económicos que buscan una explicación y una solución a su crisis.

Esta actividad manufacturera es fundamental para entender el funcionamiento de Toledo en sus aspectos económicos y sociales, por lo que es normal que sea su crisis objeto de análisis por los sectores intelectuales de la ciudad, cuyos escritos se han agrupado bajo la denominación de Escuela de Toledo.

El memorial de Juan González de Vatres Sotomayor lo podemos situar en la transición entre la ebullición que supone la Escuela de Toledo, en torno

a 1620, y los escritos posteriores a

1640. Su importancia radica en la descripción detallada de la situación

\section{ABSTRACT}

Toledo's silk industry during the Seventeenth Century is an example of a certain activity that caused a great amount of writings with the aim of finding an explanation and a solution to its crisis. This manufacturing activity is fundamental to understand Toledo's working in its economical and social aspects, therefore, it is normal that its crisis provoked an analysis from the intellectual sectors of the city, whose writings have been gathered under the designation of "The school of Toledo". The "Juan González de Vatres Sotomayor" Memorial can be sited in a transition period between the important moment that means "The school of Toledo", around 1620, and the chronicles later 1640, whose importance is in the detailed description of the situation of Toledo's industry. In

* UNED 
de la industria sedera de Toledo; en su análisis nos señala las deficiencias que sufre la sederia toledana por falta de una producción de materia prima propia, lo que marca la dependencia de Toledo con respecto a otras ciudades productoras; $y$ por una política impositiva poco flexible provocando paro y emigración y, por 10 tanto, la falta de competitividad de Toledo ante el auge de las ciudades costeras y la competencia de Madrid.

En definitiva, el memorial de Juan González de Vatres Sotomayor es un ejemplo más de la preocupación que suscita la crisis de la industria sedera en los hombres del siglo XVII toledano. the analysis are presented the deficiencies that suffers Toledo's silk industry due to the lack of its own production of raw material, which defines the dependency of Toledo with regard to other productive cities; and by a little flexible tax policy that promoted unemployment and mass departure and, consequently the lack of competitiveness of Toledo with regard to the peak of the coastal cities and the competition of Madrid. Definitively, the "Juan González de Vatres Sotomayor" Memorial is another example of the concern that caused the crisis of the silk industry among the men of Toledo in the Seventeenth Century.

La industria sedera es posiblemente uno de los recursos más importantes para el mantenimiento de Toledo en los siglos de la Edad Moderna. Su entrada en crisis tiene como consecuencia una serie de pérdidas tanto poblacionales como económicas que suponen un deterioro serio en las estructuras fundamentales del sostenimiento de la ciudad. Si bien es una industria puntera en el siglo xVI, muy beneficiada por la fluidez del comercio terrestre con Levante, en el siglo XVII el cierre discontinuo de las vias de comunicación levantinas, esenciales para el mantenimiento de la industria sedera, produce una falta constante de la materia prima, la seda cruda; también viene a coincidir con esta crisis la ausencia definitiva de la corte a finales del siglo XVI, hecho que genera una pérdida paulatina de población, no sólo cortesana sino también profesional, que emigra a otros centros más pujantes, como Valencia o Madrid. Pero además, la crisis de la industria sedera toledana no la debemos circunscribir a un nivel interno sino también en un entorno en el cual se combinan las causas nacionales como las internacionales, es decir: se va a ver afectada por el traslado de la corte a Madrid pasando a formar parte del cordón sanitario del nuevo centro político del reino, y por las fluctuaciones monetarias producto de la introducción de la moneda de vellón y su posterior consolidación como moneda de uso interno, incidiendo en las transacciones externas; y finalmente, por una guerra comercial planteada por potencias europeas como Inglaterra o las Provincias Unidas propiciando la entrada de mercaderias en la Península procedentes de las Indias Orientales. 
Esta amalgama de situaciones nos puede dar una visión de la crisis económica que se desarrollará a lo largo del siglo XVII, teniendo como referencia obligada su impacto en la industria sedera. Por lo tanto es preciso fluctuar de lo concreto a lo general, puesto que es necesario abrir la frontera de la ciudad y situarla en un contexto amplio, buscando las posibles relaciones que se pueden producir a todos los niveles, para poder analizar de forma correcta el progresivo hundimiento de una de las industrias más pujantes del entorno urbano toledano. Para conseguir este objetivo se hace necesario el análisis de toda aquella literatura económica que se produce en la ciudad en el primer tercio del siglo XVII. Este tipo de escritos es bastante abundante en la ciudad de Toledo; normalmente, con un corte industrialista, buscan soluciones para resolver la crisis de la ciudad e incluso los males del reino, como es el caso de la obra de Sancho de Moncada, posiblemente el más brillante de todos los analistas toledanos de la época ${ }^{1}$. Dentro de este grupo podemos encontrar gentes de diversas procedencias, desde el medio municipal al mercantil pasando por el universitario. Juan González de Vatres Sotomayor, cuyo memorial es objeto de nuestro análisis, procede del entorno municipal ya que se autodenomina como "jurado de esta Imperial Ciudad de Toledo, y Escrivano mayor de rentas Reales de ella" 2. Con este ejemplo vemos que la inquietud que produce el deterioro de Toledo sobre los hombres más clarividentes de la ciudad no es una cuestión aislada, sino un fenómeno continuo con una serie de propuestas más o menos brillantes y cuyo mayor desarrollo se producirá entre 1618 , fecha de la consulta realizada por el gobierno de la ciudad a la intelectualidad toledana y asimismo de la caída del duque de Lerma, y 1621, fecha de la muerte de Felipe III y subida al poder de Felipe IV y por tanto del conde-duque de Olivares, de talante más reformista al menos en sus inicios ${ }^{3}$. A pesar de que los años de

\footnotetext{
Denominación acuñada por Jean Vilar en su artículo “Docteurs et marchands: l'école de Tolède (1615-1630)", V Congreso Internacional de Historia Económica. Leningrado, 1970. Más reciente es su ponencia "Un pessimisme "calculé": l'introspection économique à Tolède (1616-1628). en Tolède et l'expansion urbaine en Espagne (1450-1650). Madrid, Casa de Velázquez, 1991. También es importante la consulta del estudio introductorio a la obra de Sancho de Moncada, Restauración politica de España. Madrid, Instituto de Estudios Fiscales, 1974, en el que Jean Vilar analiza el ambiente intelectual toledano en el primer tercio del siglo XVII.

Archivo Municipal de Toledo (AMT), Seda 1500-1650.

3 Para el caso de la actividad intelectual en la ciudad de Toledo entre 1618 y 1621 se hace obligado citar para tener una visión general la obra de CaRRobles SANTOS, J. y otros, Historia de Toledo. Toledo, Azacanes, 1997, en su parte dedicada a la crisis del siglo xvil; y de modo más concreto el artículo de ARANDA PÉREZ, F.J.: "La preocupación "arbitrista" en el seno del ayuntamiento de Toledo por la declinación de la ciudad en un período crítico: 1618-1621", Toletum, núm. 29, 1993. Págs. 201-227, en el cual se nos analiza el movimiento intelectual que se produce en los años de referencia, y la ponencia de Rodriguez DE Gracia, H.: "Alonso de Castro
} 
máxima producción los debemos situar entre 1618 y 1621, no podemos olvidar que las consecuencias de esta importante creación arbitrista en la ciudad de Toledo se pueden rastrear en años posteriores, tomando como punto de inflexión la década de los años treinta del siglo XVII, período un tanto oscuro en el que la calidad de las propuestas empieza a bajar de forma ostensible.

En definitiva, nos encontramos con una situación en la que la crisis es patente en amplios sectores productivos, no sólo de la ciudad de Toledo sino también en otros centros peninsulares. El sector sedero en especial se ve afectado por causas endógenas, como las crisis de subsistencia agravadas por las oleadas de enfermedades que afectan al tráfico interno; y por el uso de una moneda de valor inferior cuya utilización en el exterior es imposible. Por otra parte, es reseñable la pérdida de población que sufre Toledo, afectando de forma clara a sus sectores productivos, incluyendo por supuesto la industria sedera, que asimismo se verá perjudicada por la guerra comercial planteada por potencias como Inglaterra o las Provincias Unidas y que provoca la entrada de productos de menor calidad pero más asequibles en sus precios, lo que facilita que una importante parte del capital se desvie a zonas tan dispares como Europa o China ${ }^{4}$. A ello hay que añadir la menor entrada de metales preciosos procedentes del Nuevo Mundo, unida a la escalada del contrabando y a las injerencias continuas, en forma de piratería, de las potencias comerciales opuestas a la Monarquía Hispánica. La conocida crisis de 1620 tiene consecuencias fundamentalmente económicas y estructurales, debido al ataque de las bases monetarias de la corona y a un recrudecimiento de los problemas internos del país tanto políticos como económicos o poblacionales. De este modo las propuestas de Juan González de Vatres Sotomayor, a pesar de ser de fecha posterior a los años más candentes, reflejan toda aquella amalgama de situaciones negativas que afectan muy directamente al sector sedero toledano, tomando en algunos casos una dimensión ex-

Xibaje: un arbitrista del siglo XVII', en Tolède et l'expansion urbaine en Espagne (1450-1650). Madrid, Casa de Velázquez, 1991, en la que se nos muestra un caso concreto de un arbitrista toledano de la época. Sobre el talante reformista del conde-duque de Olivares baste citar la obra de ELLIOTT, J.H.: El conde-duque de Olivares. El político en una época de decadencia. Barcelona, Crítica, 1990.

4 Sobre este punto hay que señalar los comentarios expuestos por Pierre Vilar en su artículo "Los primitivos españoles del pensamiento económico. "Cuantitativismo" y "Bullonismo", re-

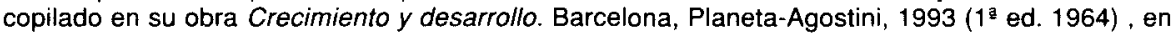
el cual sitúa este hecho a fines del siglo xvI. También es interesante consultar los memoriales de 1616 de los reinos de Granada, Valencia y Murcia sobre los daños que causa la importación de seda de reinos externos a España localizables en A.M.T., Seda, 1500-1650, movimiento al que se une también el reino de Toledo. 
terna, ya que las causas del declive del sector que describe pueden ser extrapolables y explicadas por razones ajenas a la ciudad. También se hace necesario señalar que la fijación de la corte y el trasiego humano hacia Madrid se convierte en recurrente en muchas de las propuestas que se exponen al ayuntamiento de la ciudad de Toledo por muchos arbitristas, motivo que no está ausente en las propuestas de Juan González de Vatres Sotomayor.

\section{CARACTERISTIICAS TEÓRICAS DE LOS ESCRITOS ECONÓMICOS TOLEDANOS}

Para poder sistematizar el desarrollo de este punto se hace obligatorio citar en primer lugar las palabras de Francisco José Aranda Pérez sobre el caso de Toledo:

Esta imperial ciudad se muestra aparentemente como una de las partes más afectadas de toda la monarquía española, o, al menos, como uno de los lugares que más pronto conocen la gran crisis que se avecina y que claramente se empezaba a barruntar desde los últimos años del siglo XVI. El marcado contraste del difícil presente con un pasado más o menos esplendoroso (en el Quinientos) provoca que en el seno de la ciudad se reflexiona de manera profunda sobre la crisis, sobre sus causas y consecuencias, llegándose con ello a una serie de conclusiones y fórmulas - más o menos sensatas - que no sólo van a tener incidencia en el pensamiento local, sino que también nutrirán en general, a través de lo que se ha llamado la Escuela de Toledo, el análisis económico arbitrista de la crisis del conjunto del Reino ${ }^{5}$.

En general la obsesión del grupo toledano es la entrada de mercaderías extranjeras, circunstancia que denuncian con un talante claramente prohibitivo. Los escritos de los teóricos toledanos tienen un carácter fundamentalmente industrialista, ya que la producción manufacturera es considerada como el motor económico de la ciudad; de ahí que la mayoría de las soluciones que se proponen sean de tipo proteccionista en torno a este sector. En general los arbitristas toledanos consideran la industria como un productor de importantes recursos no sólo para la ciudad, sino también para la propia corona, gracias a la recaudación de impuestos ${ }^{6}$.

\footnotetext{
5 ARANDA PÉREZ, F.J.: “La preocupación "arbitrista" en el seno del ayuntamiento de Toledo por la declinación de la ciudad en un periodo critico: 1618-1621", Toletum, núm. 29, 1993, pags. 202-203.

6 Este aspecto es analizado en la obra de PERDICES DE BLAS, Luis, La economía política de la decadencia de Castilla en el siglo xvil. Madrid, Síntesis, 1996.
} 
Para poder beneficiarse de este potencial, se hace necesario un proteccionismo claro y sin concesión ante la entrada de productos extranjeros; y es posiblemente éste uno de los caracteres definitorios del grupo toledano ${ }^{7}$.

En muchos casos los escritos teóricos de los discursos confeccionados por los arbitristas toledanos tenían el objetivo principal de ser también prácticos, es decir, los memoriales no eran meros escritos utópicos que tenían una sólida base teórica sino que aportaban innumerables soluciones que, a su parecer, podían ser llevadas a la práctica de una manera racional. De ahí la importancia de aportaciones, como las de Juan Belluga de Moncada, de corte claramente proteccionista, o las de Sancho de Moncada, bien a través del memorial de la Universidad de Toledo, en el que se resumen gran parte de sus ideas ${ }^{8}$ o en su obra cumbre Restauración política de España, que transciende más los límites de la propia ciudad. A esta obra cabe añadir las aportaciones de Damián de Olivares, cuyo interés radica en su procedencia mercantil y en que en gran parte refleja el sentir del sector de los mercaderes. En el caso concreto del autor objeto de nuestro estudio, las características generales antes señaladas se cumplen en casi todos sus extremos; pero además sus propuestas están íntimamente relacionadas con la industria sedera ${ }^{9}$, la cual describe en todos sus procesos productivos y comerciales internos. A pesar de corresponder a una fecha más tardía al momento de ebullición de la inquietud introspectiva económica de la ciudad - se trata de un escrito fechado en 1636-, las características son semejantes. Es evidente que este memorial no alcanza las cotas de calidad o de lucidez de las obras de Sancho de Moncada o de Olivares, pero su importancia estriba en que el análisis que realiza Juan González de Vatres Sotomayor de los males de la industria sedera es una fuente de primera mano para poder valorar cuál era la importancia de las labores de seda en Toledo y, en cierto modo, revela que nos encontramos ante una de las industrias más

\footnotetext{
7 El carácter proteccionista de los escritos toledanos queda reflejado en las obras de RooRiGUEZ DE Grac|A, H.: "Alonso de Castro Xibaje: un arbitrista del siglo XVII», en Tolède et l'expansion urbaine en Espagne (1450-1650). Madrid, Casa de Velázquez, 1991, y en la de CARROBLES SANTOS, J. y otros Historia de Toledo. Toledo, Azacanes, 1997, en su punto dedicado a la producción arbitrista toledana del siglo xVII. De igual modo este carácter se puede detectar de forma clara en la documentación original que se analizará a continuación.

8 Este memorial se puede consultar en el Archivo Municipal de Toledo, al igual que la carta que remite el propio Sancho de Moncada reconociendo la autoría del memorial. En este último caso véase A.M.T. Cartas. Año 1620. También es obligada la consulta de su obra Restauración política de España, ed. de Jean VILAR. Madrid, Instituto de Estudios Fiscales, 1974.

9 La defensa de la industria sedera toledana está presente en todos los escritos económicos importantes de la época realizados en la ciudad de Toledo.
} 
importantes de la ciudad, ya que es considerada como uno de sus motores fundamentales.

Fundamental es también el aspecto demográfico en las razones que argumentan los arbitristas toledanos a la hora de valorar la creciente decadencia que sufre la ciudad en el primer tercio del siglo XVII. Esta es una de las preocupaciones primordiales de los teóricos, puesto que la pérdida de población, debida en gran parte al traslado de la corte, afecta de forma clara a los sectores productivos de la ciudad y por tanto a la recaudación de impuestos necesaria para el mantenimiento de Toledo. Casos como el de Juan Belluga de Moncada ${ }^{10}$, que solicita la vuelta a Toledo de las personas que se trasladan a la corte por su importante poder adquisitivo, no son nada aislados. Si nos remitimos al memorial que presenta la Universidad de Toledo al rey en 1620, podemos encontrar argumentos que se basan en la despoblación para explicar la crisis no sólo de Toledo sino incluso a nivel peninsular. Sirvan las siguientes líneas como ejemplo:

...que de aqui a resultado la general despoblacion de España, que ha sido muy grande de pocos años acà, sin aver en ellos las causas que algunos dan de ellas. La razon es tan patente, como las demas: porque la pobreza a impedido la procreacion y la conservacion de la gente: $y$ esto se vee por mayor, que no ay el diezmo de casamientos ni de bautismos en ciudades y lugares que solian tener fabricas de mercaderias, que en los que solian vivir de su labrança, y que estan mas assolados los lugares de comarca de las ciudades que fabricavan mercaderias, que los que estan en comarca de ciudades que nunca las han tenido ${ }^{11}$.

Al igual que Sancho de Moncada, otros autores también encuentran en la despoblación de las ciudades productoras de artículos industriales un signo inequívoco de la decadencia que sufre el país. El caso de Toledo es extremadamente significativo, ya que cumple en todos sus puntos los condicionantes negativos expuestos en el memorial de la Universidad de Toledo. La despoblación tiene como consecuencia inmediata el colapso de las actividades manufactureras, con el consecuente debilitamiento del mer-

to Sobre los memoriales de Juan Belluga de Moncada son recomendables los análisis realizados en la obra de CarRobles SANTOS, J. y otros, op. cit. y en el artículo de ARANDA PÉREZ, F.J.: op. cit., en el que se expone que Belluga de Moncada no ejerce el papel de pensador económico sino más bien el de transmisor y portavoz en la corte de este tipo de temas dada su condición de jurado de la ciudad de Toledo. Asimismo uno de los memoriales de Belluga de Moncada está transcrito y publicado por Antonio Dominguez OrTiz en su obra La sociedad española del siglo XvII.

11 La Universidad de Toledo suplica en primer lugar a V.M. en la gravisima junta. No salgan de España materiales laborables, ni entren mercaderias labradas fuera de ella, ò por lo menos no entren las que se pudieren escusar... A.M.T. 
cado. De este modo, la inevitable regresión de los ingresos impositivos en las ciudades provoca que las continuas inflaciones y deflaciones de la moneda de vellón afecten de forma considerable al entorno urbano. Para evitar esta serie de efectos negativos sobre la economía urbana se hace necesaria una política proteccionista para impulsar la producción y distribución del producto interno $y$, de paso, evitar la paulatina despoblación de importantes ciudades productoras como Toledo. En definitiva, la continua fluctuación monetaria provoca prácticas ilícitas como la especulación, que produce un ambiente irrespirable tanto a oficiales como a mercaderes, lo cual provoca una huida hacia otras ciudades más propicias a sus intereses, el abandono y degradación del entorno urbano en trance de despoblación y la ruina de los que se quedan.

\section{ANTECEDENTES DEL MEMORIAL DE JUAN GONZÁLEZ DE VATRES SOTOMAYOR}

A la hora de fijar las bases del memorial de Juan González de Vatres Sotomayor, debemos referirnos obligatoriamente a una serie de escritos de tipo proteccionista que marcan muchas de las claves que describen el sentir económico en el primer tercio del siglo XVII, no sólo de la ciudad de Toledo sino también de otros reinos que tienen como nexo común la producción sedera, tanto a nivel transformador como productor de materia prima. De este modo podemos ver cómo evoluciona el proceso de decadencia de la industria sedera toledana en beneficio de otros focos que muestran un claro rechazo a la entrada de productos extranjeros, a lo que se adhiere el reino de Toledo; por otra parte, también podemos analizar los intentos legislativos de proteger la producción sedera toledana mediante medidas coercitivas sobre la tenencia de telares fuera de un límite fijado alrededor de la ciudad de Toledo y sus arrabales.

En el caso de los memoriales de los diferentes reinos productores de seda, hay que significar que se trata de una serie de escritos dirigidos al rey en 1616 para evitar la entrada de seda en bruto procedente de lugares de Extremo Oriente, como China o la India. Posiblemente la explicación más interesante para negar la entrada de seda oriental en la península, que exponen los reinos de Granada, Murcia y Valencia, sea la siguiente:

Que siendo estos Reynos de España, como son, tan faltos de oro, y de plata, que solo tienen el que se ha traydo y trae de las Indias, y otros Reynos estraños, con los frutos y esquimos que en estos se cogen siendo uno de los muy principales este de la seda, y que del se proveen los otros Reynos, parece conveniente que se conserve su precio y estimacion, pues con el causa 
que do se crian se sustenten con sustancia para poder acudir al Real servicio en las ocasiones que se ofrecen. la gente pobre con la ocupacion del se remedie los lugares do se labra y texe, hagan lo mismo, que si faltasse el dicho precio y estimacion, todo daria una gran baxa ${ }^{12}$.

Como vemos, nos encontramos ante una situación en la que convergen diferentes matices a la hora de analizar el sentimiento de crisis que se expresa en estos memoriales. Por un lado, es patente que la falta de recursos monetarios debida a la disminución en la entrada de metales preciosos provoca una menor competitividad de los productos sederos, al producirse la bajada de los precios según la ley cuantitativa: esto repercute de forma negativa porque los productos venidos de fuera son aún más baratos y de menor calidad. Así nos encontramos con una disminución ostensible de la producción, lo que perjudica a los ingresos de la hacienda real; $y$, a su vez, los recursos monetarios se desvían a zonas fuera del reino, debido al intercambio comercial que produce la entrada de estos productos provenientes de Extremo Oriente. Pero esto no es nuevo: como nos señala Pierre Vilar, a finales del siglo XVI la falta de protección que sufre la industria provoca que el dinero se desvíe a otras zonas, debido a una competencia ante la cual el producto español no tiene capacidad de respuesta ${ }^{13}$. Pero a esta situación se une una necesidad suntuaria de la propia corte, que genera importantes gastos dentro del Estado, con consecuencias negativas a la hora de intentar proteger a la industria, ya que la introducción de la moneda de cobre para uso interno repercute en la menor fluidez de la moneda de metal precioso. Si continuamos con Pierre Vilar, se puede afirmar que

"las costumbres suntuarias de los grandes, los enormes gastos del Estado, la generalización de la deudas no se lo permitan. Recibiendo menos moneda buena y teniendo que enviarla al exterior, España fabricará otra mala para uso interno: con el siglo empieza la gran acuñación de cobre, la má quina de hacer billetes de la época. Entre 1600 y 1610 las Cortes y los teóricos monetarios, criticando sin cesar esta política, predicen la catástrofe" 14

Pero no sólo se trata de una simple crisis comercial sino de una desestabilización estructural debida a la devaluación del sistema monetario interno, que repercute en la competitividad industrial de las ciudades es-

\footnotetext{
12 A.M.T., Seda, 1500-1650.

13 Véase el artículo de VILAR, Pierre, "Los primitivos españoles del pensamiento económico... op. cit. En el cual se expone esta idea.

${ }_{14}$ VILAR, P: "El tiempo del "Quijote"». Crecimiento y desarrollo. Barcelona, Planeta-Agostini, 1993, pág. 335.
} 
pañolas. Es evidente que el sector textil se ve afectado de forma directa por la falta de moneda solvente y también lo es que el sector sedero, muy localizado en determinados puntos, se ve bloqueado por la injerencia comercial de otras potencias que promueven la introducción de estas sedas orientales objeto de la protesta. A esta preocupación, introducida en el seno de la corona por los reinos de Granada, Murcia y Valencia, se une la de otras ciudades como Córdoba y la provincias de Valladolid y Salamanca. EI apoyo de Toledo es indudable. Este movimiento produce la presentación en la corte de nuevos memoriales en los que se describe la productividad de la seda y los beneficios que pueden acarrear a la Real Hacienda. Sirva como botón de muestra la descripción que en el memorial de 1616 se hace de la bajada en la recaudación impositiva en los diferentes reinos a causa de la menor producción, fruto de la entrada de seda extranjera:

En el Reyno de Granada terna la Real hazienda de V. Magestad con la falta de este esquimo, una gran quiebra, por rentar como renta en los derechos de dicha seda, quarenta y cinco quentos, que estos, y todos los censos perpetuos a que estan dadas las haziendas, y poblaciones de aquel Reyno, cessaran, y se despoblara, por no poder sustentar en el: y en los de Valençia, y Murcia, do asimismo su principal sustancia es el dicho esquimo: y en los de Toledo, Sevilla, Cordova, y laen, y otros, do se fabrica y texe dicha seda (que causa en cada uno de ellos el mayor miembro de alcavala) ternan tan gran quiebra, que no solo no se podra hazer segundo assiento en el cabeçon general, pero con mucha dificultad se podra cumplir el tiempo que del que corre queda, y los situados sobre dichas alcavalas no se podran pagar: cosas todas tan considerables, que piden particular remedio ${ }^{15}$.

Está claro que los diferentes reinos ven disminuir sus ingresos debido a la entrada de mercaderías externas, lo cual deteriora de forma ostensible al entorno urbano. La disminución de la recaudación de la alcabala de la seda en el caso de Toledo provoca una regresión considerable del erario municipal porque la actividad sedera es posiblemente una de las más importantes de la ciudad. El carácter de Toledo como ciudad importadora de seda en bruto - Toledo no es productora de la materia prima debido a diferentes litigios de propiedad con hermandades y sobre todo con la iglesia-, hace necesario el mantenimiento de un comercio activo con las zonas productoras, especialmente Valencia y Murcia. Esta actividad comercial supone unos importantes ingresos impositivos para el ayuntamiento en forma de derechos de portazgo. Además, la fluidez de la entrada de la seda en bruto mantiene en operatividad todos los oficios que componen el sector sedero, como son los torcedores, los tintoreros y los

15 A.M.T., Seda, 1500-1650. 
tejedores. La respuesta de la ciudad de Toledo, como se ha repetido constantemente, es clara a favor de los intereses de las ciudades productoras de seda, y por tanto, favorable al mantenimiento de su comercio. Para tal fin el ayuntamiento nombra a una serie de comisarios para que den su aprobación a los memoriales promovidos por los reinos de Granada, Murcia y Valencia, obteniendo como respuesta lo siguiente:

...por todas las rrazones que dizen en su memorial y particularmente por que es cosa çierta y llana que conviene mucho el sustentar en estos rreinos el trato de la seda y cria della por y de que depende el sustento de ynfinita gente y si se da lugar a que entre seda de fuera del rregno no es posible conservarse en estos rreinos la crianza de la dicha seda porque es cosa llana que se perderan los moreros y las personas que tratan en este trato... ${ }^{16}$.

En definitiva, nos encontramos ante una injerencia comercial de grandes dimensiones orquestada tanto por Holanda como por Inglaterra. La menor afluencia de metales preciosos provocada por la intervención de las potencias comerciales contrarias a la corona hispana y el patrocinio de la introducción de materias como la seda en la península, bien a través de las rutas del Mediterráneo, o por el puerto de Lisboa, provocan el comienzo del colapso de la actividad manufacturera española. A esto debemos añadir una política interna de endeudamiento progresivo del Estado. La ostentación y corrupción de las clases dirigentes hacen un flaco favor a los intereses de la corona, a lo que se une la creación de una moneda de uso interno sin ningún valor en las transacciones comerciales exteriores, puesto que estas últimas se deben realizar con moneda de plata, y ello provoca la desviación de los metales preciosos provenientes de América hacia el exterior de las fronteras nacionales, junto con el pago de los innumerables préstamos realizados por banqueros extranjeros.

A consecuencia de la presión ejercida por la ciudad de Toledo en defensa de su sector sedero, en 1619 se consigue la promulgación de una cédula real mediante la cual se prohibe la tenencia de telares de seda en un contorno de quince leguas a la redonda de Toledo. Por tanto, los telares debían estar obligatoriamente situados en la ciudad y sus arrabales. Con esta cédula se intenta el mantenimiento de una forma forzada de la industria sedera en el entorno urbano para evitar, de este modo, la fuga de artesanos a pueblos de alrededor y la producción sedera en pueblos teóricamente dependientes de Toledo. A pesar de este carácter proteccionista, también encontramos que la promulgación de esta cédula real es moneda

16 Ibidem. 
de cambio a los servicios prestados por la ciudad a la corona. En las siguientes líneas se demuestra lo anteriormente expuesto:

Por quanto por parte de Vos el ayuntamiento y corregidor de la ciudad de toledo nos a sido echa relacion que entre otras condiciones que os emos conzedido en considerazion de haver venido por Vuestro boto desçesibo en el servicio de los diez y ocho millones con que el reyno nos a servido por el consultibo de sus ciudades y Villa, es que no se pueda tejer ningun genero de tegidos de seda de qualquier suerte e manera que sea quinze leguas en contorno de toledo sino solamente en ella y sus arrabales, por ninguna causa ni razon que sea en poca, o mucha cantidad... ${ }^{17}$.

De todos modos, a pesar del intercambio de favores, lo realmente interesante es analizar el calibre de la medida proteccionista que supone esta cédula real del 8 de junio de 1619. La limitación de la tenencia de telares supone la eliminación de la competencia que puede suponer una producción sedera en los pueblos de los alrededores de la ciudad, con unas condiciones impositivas diferentes según la condición de la población. En este punto se añade un elemento interesante: la importante relación de intercambio existente entre el entorno urbano y el rural. Este último, en materia manufacturera, debe ser en principio dependiente de la ciudad, más en un producto tan competitivo como el de la seda. La implantación de telares en los pueblos cercanos no supone que el entorno rural produzca para el urbano sino que el ámbito rural compite con la ciudad, lo cual provoca que se adopten medidas proteccionistas a este respecto. Además, la implantación de telares en las zonas rurales determina el desvío de las rutas comerciales que, en muchos casos, tienden a dirigirse a estas zonas para evitar el sistema impositivo de la ciudad, y, en casos extremos, evitar el cordón sanitario del que forma parte Toledo en épocas de epidemia para proteger a la corte. La importancia de esta medida la podemos calibrar teniendo presentes las penas que establece en caso de incumplimiento:

...que el que contrabiniere a ello por la primera vez que fuere denunciada caya e yncurra el tejedor, o tejedora en perdimiento del telar aunque no sea suio y quatro años de destierro y del lugar donde fuere denunciado y diez mill maravedis y el dueño de la seda en perdimiento della, con otro tanto repartido en quatro partes, la primera para nuestra camara, la segunda para el juez que los sentenciara la terçera para el denunciador y la quarta y hultima para la dicha ciudad de toledo con que lo desto proçediere aya de servir y sirva para aumento de las ventas de las nuestras alcavalas de la dicha ciudad... ${ }^{18}$. 
El talante de intercambio de intereses sigue presente en el texto legal debido a que el soporte que supone para la corona la aportación de las ciudades es bastante importante. Mediante la limitación de la tenencia de los telares a quince leguas a la redonda de Toledo también se pretende afianzar la recaudación impositiva de la ciudad para así tener los recursos suficientes y necesarios para la corona. Esta medida real nos demuestra la importancia que tiene la producción sedera en la ciudad. Como se ha señalado con anterioridad, la inmensa mayoría de los pensadores económicos toledanos sostienen que la industria sedera es una de las bases económicas de la ciudad de Toledo. En conclusión, nos encontramos con una medida proteccionista en la que el intercambio de intereses entre la corona y la ciudad es patente. Este intercambio denota una necesidad de controlar los recursos impositivos para favorecer en última instancia los intereses de la corona, y para tal fin se hace necesaria la creación de una figura burocrática que vele por el cumplimiento de la orden real. La cédula contempla el nombramiento de un juez particular que atienda a las causas referidas en la cédula real; esta nueva figura también es objeto de controversia, ya que intervienen intereses personales en su nombramiento, lo cual queda nítidamente reflejado en una carta dirigida por el ayuntamiento toledano al rey:

Entre las mercedes que $V$. magestad fue servido de hacer a esta ciudad en causa de relacion del servicio de los diez e ocho millones fue una de dar su rreal cedula para que en quinze leguas en contorno desta ciudad no se pudiesse texer ni fabricar ningun genero de sedas sino fuese en ella y sus arrabales y que en cualquier tiempo que esta ciudad pidiesse juez particular para la observancia de lo susodicho se le diesse y señalase y esta merced hizo $\mathrm{V}$. magestad a esta ciudad para que usasse della cuando y como les conbiniesse conforme a las ocassiones que se ofreziesen y siendo esto anssi avemos tenido notiçia que Juan belluga jurado de esta ciudad sin horden ni comission nuestra a tratado de pedir se nonbre juez para el cumplimiento de esta merced... ${ }^{19}$.

En este caso nos encontramos con una acción interesada de nada más y nada menos que Juan Belluga de Moncada, al cual hemos destacado como figura importante de la llamada Escuela de Toledo. Con esta acción el ayuntamiento bloquea el cumplimiento de la orden real y deja vacante la figura del juez al no aceptar la injerencia de Juan Belluga de Moncada que, a la sazón, es jurado ante la corte en representación de Toledo. El nombramiento regio de Juan Belluga de Moncada como juez particular

\footnotetext{
19 Ibidem.
} 
para el cumplimiento de la cédula real de las quince leguas provoca una reacción airada del consistorio, puesto que se ve desposeído de las funciones reflejadas en la propia cédula. De este modo el ayuntamiento considera que el nombramiento de Juan Belluga «tiene muy grandes ynconbinientes especialmente que siendo merced hecha a esta ciudad para que use della como y cuando mexor le pareciere que conbiene no es justo que sin su horden se trate de executar" ${ }^{20}$. Pero si explicamos como un juego de intereses personales la reacción del ayuntamiento, caeríamos en lo puramente anecdótico. Esta situación marca uno de los efectos propios de un momento de crisis, el oportunismo. La acción personal de Juan Belluga denota bastante ambición; pero con esta reacción, el ayuntamiento toledano por su parte demuestra que no quiere perder el control sobre lo que considera sus bienes, y para ello argumenta que ya hay un exceso de cargos burocráticos, lo cual aumenta el coste de las diligencias, y que el nombramiento de este juez particular también supone unos honorarios que la ciudad entiende injustificados. Este tipo de hechos demuestra la precaria situación en la que se encuentra la hacienda municipal porque el ayuntamiento prefiere esperar a que los casos de incumplimiento de la cédula sean claros, para después actuar sin coste alguno. Por esta razón ues muy grande ynconbiniente aber juez nonbrado en essa corte porque se entiende que el dar libertad para pedir este juez quando le conbiniere y cuando se tubiere noticia que en algun lugar se contrabiniere a esta merced o el gremio quando ubiesse menester juez lo pidira sin tener salario ni costa ordinaria» ${ }^{21}$. Esto parece que va en contra de los intereses de Juan Belluga.

En definitiva, con estos dos ejemplos documentales, antecedentes de las propuestas de Juan González de Vatres Sotomayor, nos encontramos con dos hechos puntuales que marcan a nivel externo e interno la situación en la que se encuentra la industria sedera en las fechas cercanas a la crisis de 1620. Como vemos, los hechos que se describen son bastante concretos y no podemos considerarlos como memoriales arbitristas propiamente dichos; pero la importancia que tiene pulsar el sentir de las ciudades productoras ante la competencia extranjera y el intercambio de favores a través del mantenimiento del sistema impositivo de forma bastante débil, se nos revelan claros ejemplos de una situación bastante generalizada en el primer tercio del siglo XVII. Como nos confirma Hilario Rodríguez de Gracia, los momentos deflacionistas se dejaron ver en el empobrecimiento de las masas campesinas, la despoblación, el colapso artesanal,

20 Ibidem.
21 Ibidem. 
las alteraciones del vellón, el debilitamiento de la actividad mercantil, aparte de otros fenómenos que por no mencionados dejaron de tener importancia" ${ }^{22}$. En cierto modo los dos hechos referidos confirman esta situación.

\section{EL MEMORIAL DE JUAN GONZÁLEZ DE VATRES SOTOMAYOR}

Como hemos visto en los puntos anteriores, la situación de la ciudad de Toledo no es nada alentadora en lo que se refiere a la producción manufacturera. Dentro de ésta, la industria sedera es posiblemente una de las más afectadas por la situación de crisis. En 1636, año en que Juan González de Vatres Sotomayor realiza sus propuestas para la salvación de la industria sedera, la crisis ya no solo actúa en contra de las bases productivas de la ciudad sino también en la propia estructura social. La despoblación producida por el traslado de la corte es patente, lo cual nos indica una pérdida ostensible de la mano de obra cualificada; a su vez el anquilosamiento de los mecanismos impositivos propicia la marcha de los comerciantes hacia otras zonas destacando el traslado humano hacia la corte. Por otra parte, el desarrollo de industrias sederas en otras ciudades como Valencia provoca que las zonas productoras de seda en bruto desarrollen su mercado con un potencial comercial más alto que el de Toledo debido a su salida al mar y su proximidad, con lo que las rutas comerciales terrestres se ven seriamente afectadas ${ }^{23}$. Nos encontramos por tanto ante un proceso de competencia por parte de la corte y de un desmantelamiento progresivo de los canales comerciales que surten a la industria sedera de la materia prima necesaria para su desarrollo, pues Toledo no es una ciudad productora de seda en bruto.

El caso de Juan González de Vatres Sotomayor es fiel reflejo de la preocupación existente dentro del seno de los cargos representativos de la ciudad toledana. Como ya se ha indicado, Juan González es jurado de la ciudad de Toledo y además escribano mayor de rentas reales, por lo que podemos deducir que su conocimiento de los movimientos impositivos de la ciudad es bastante amplio. También hay que señalar que su memorial está dirigido al ayuntamiento y no al rey, lo cual indica que en 1636 se mantiene la preocupación del consistorio ante la crisis estructural que sufre

22 Rooriguez de Gracia, $\mathrm{H}$.: "Alonso de Castro Xibaje: un arbitrista del siglo xvil», en Tolède et l'expansion urbaine en Espagne (1450-1650). Madrid, Casa de Velázquez, 1991, pág. 99.

23 Sobre este tema es recomendable la consulta de la obra de CASEY, James, El reino de Valencia en el siglo xvii. Madrid, Siglo xxl, 1983, sobre todo en los capítulos dedicados a la decadencia de la agricultura y a la actividad comercial. 
la ciudad, si bien de forma más débil que en el momento de apogeo de la actividad teórica que representan los años inmediatos a 1620. Al iniciar el análisis del memorial de Juan González de Vatres Sotomayor, es necesario citar sus argumentaciones para justificar la defensa de la industria sedera toledana, explicando a su vez todo los procesos y subsectores que la componen:

Lo primero que el principal comercio, trato y grangeria de esta Ciudad, y donde depende la conservacion de ella es la fabrica de la seda, que se cria en el Reyno de Valencia, y en Murcia, y se trae a esta ciudad en cruda, donde se beneficia por manos de Torcidores, que sustentan mucha gente pobre, a quien la dan a devanar, de donde viene al Mercader que lo comprò, y en su casa con su familia, y otras personas lo adereçan, y entregan a Tintoreros para que lo tiñan de las colores necessarias, los cuales ocupan a mucha gente, y de alli lo buelven a entregar al dicho Mercader, que haze muchas suertes, y entrega a mugeres de todos estados para que lo devanen, y pongan en cañones, y açarxas, y de ellas buelve al dicho Mercader, que lo dà a urdir, $y$ hazen telas, y medias a otras personas; y sacado deste urdido, lo entrega a Texedores para que texan estas telas, y listoneria: y estos Texedores tienen sus casas, y familias, que todos se ocupan en disponello, como es en tirar, aviar, hazer canillas, y otras cosas forçosas, y necessarias. $Y$ todo este genero de gente es mucha en esta Ciudad, y en tanto grado, que de las tres partes son mas de las dos, y los que gastan el vestuario, carnes, y frutos de la tierra; y si este trato cessa en esta Ciudad, està acabada totalmente ${ }^{24}$.

La importancia de la industria sedera no estriba simplemente en su producción y en los recursos económicos que puede generar sino también en la cantidad de personas que emplea y que por tanto son consumidores de productos de primera necesidad. Como vemos en las argumentaciones de Juan González, el proceso de fabricación de un tejido de seda mueve a gran cantidad de personas implicando la acción de tres oficios de forma escalonada: los torcedores son los primeros que intervienen en el proceso, ya que son las personas que transforman la seda en bruto en hilo; posteriormente los tintoreros dan color a este hilo, con lo que queda preparada la seda para ser tejida y ser transformada en paños $u$ otras prendas, función que realizarán los tejedores. En el texto también queda bastante clara la función del mercader, que es la figura que trae la seda a la ciudad y posteriormente es el nexo de unión entre los oficios que componen el sector sedero. Con este trasiego necesario de la seda de un lugar a otro se puede afirmar que el propietario de la materia prima y posteriormente del producto final es el

24 A.M.T., Seda, 1500-1650. 
mercader que utiliza y paga los servicios de los maestros de los distintos oficios sederos.

Según las palabras de Juan González de Vatres, la industria sedera es la más importante de la ciudad. No cabe duda de que es un sector del que depende gran parte de la población, al fabricar un producto bastante competitivo en la época, ya que se trata del material utilizado en la tela de los trajes de las clases privilegiadas; también la seda es componente de otras prendas como medias, bonetes o cintas. Nos encontramos por tanto ante un sector importante pero a su vez débil por la dependencia que supone el tener que importar la seda en bruto de otros puntos de la península, lo cual está sujeto a las fluctuaciones de la moneda de vellón, a la situación sanitaria del país y a prácticas ilícitas como la especulación. Toda esta amalgama de inconvenientes incide de forma negativa en la producción porque hace subir los precios a medida que falta la materia prima, haciendo disminuir los ingresos de los artesanos al bajar su productividad. Esta falta de recursos repercute en la propia vida de la ciudad de forma negativa al bajar el poder adquisitivo de un importante sector de la población, contingente humano que también tiene una clara tendencia a trasladarse a otros lugares buscando mejor fortuna. Juan González de Vatres Sotomayor nos explica estos movimientos negativos del siguiente modo:

Y el año que falta el entrar en ella (en la ciudad) seda en abundancia, se reconoce, porque los Mercaderes, y los hombres de negocios que tienen este trato, si les falta de que hazer la fabrica, como tienen tantos gastos, y costas en sus casas, se pierden, y quitan el dar en que entienda toda esta gente, que ordinariamente los mas pobres, assi hombres, como mugeres, los unos cargados de hijos, otras viudas, y donzellas, que de afligidos, como les falta el sustento, se van a otras partes a usar de diferentes oficios, y dexan sus casas desamparadas; y por ventura otros dan en vicios temerarios ${ }^{25}$.

Estos momentos de desabastecimiento producen movimientos bruscos dentro de la población productiva. La fuga de la mano de obra tiene efectos negativos en el mantenimiento de la propia ciudad al aumentar el abandono de las casas, que entran en un estado de ruina temprano dejando un desolador paisaje en el entorno urbano. Esto también supone una menor fuente de ingresos para el ayuntamiento. $Y$ progresivamente Toledo, de forma inexorable, va quedando en manos de la única institución viva dentro de la ciudad, la iglesia. Por tanto el paulatino desmantelamiento de la industria sedera toledana nos da una de las claves fundamentales de la decadencia de Toledo, que pasa de ser una ciudad puntera en el siglo XVI a

25 A.M.T., Seda, 1500-1650. 
ser un lugar de segundo orden en poco más de un tercio de siglo. La falta de esta población repercute a su vez en otros sectores dependientes y en el propio sistema impositivo de la ciudad ya que al faltar las rentas de estos habitantes la ciudad cae en un estado de ruina no sólo física sino también económica, lo cual se verá en la imposibilidad en muchas ocasiones de poder afrontar con solvencia las obligaciones que para con la corona tiene adquiridas Toledo. A esto se añade el arcaísmo del sistema impositivo toledano que hace poco atractivo el comercio de la seda entre Toledo y Levante. Según Juan González de Vatres Sotomayor, la crisis que sufre la ciudad es debida en gran parte a que no viene seda de Valencia y Murcia por la gran cantidad de impuestos a los que está sometida, con el consiguiente desvío a otros puntos más favorables, donde incluso se han trasladado los propios artesanos toledanos, que mantienen sus formas de elaborar el producto final. En palabras del jurado toledano, "en esto es necessario poner remedio, porque sino, se acabarà de hundir la ciudad, y la Real hazienda va cada dia en diminucion, como lo hemos visto en sus alcavalas" ${ }^{26}$. El recuento que hace Juan González de Vatres Sotomayor es cuando menos clarificador:

...el año passado de 1628. (la alcabalas) valieron mas de 54. quentos, con que su Magestad pagò, y satisfizo al situado de juros que tiene sobre estas alcavalas, y le sobrò un gran pedaço de dineros, y oy serà harto que valgan 40 . quentos, y cada año han de valer mucho menos ansi ellas, como las atavas de vino, vinagre, y azeite, sisas de carnes, por no aver gastadores: y los Arrendadores que arrendan las rentas, se pierden en ellas, contenellas en mas moderados precios que los que pierden en ellas, (sic) con tenellas a mas moderados precios que los años passados. $Y$ para que mejor se verifique esta renta de sedas, que es la mas abundante, y de mas consideracion, que valia su alcavala 12. quentos de maravedis, antes mas que menos, baxò el año passado de 1633. a 8. quentos, poco mas o menos, y no por mala administracion, ni porque dexan de pagar lo bastante los contribuyentes en ella, sino por falta de las entradas de la dicha seda; donde se caussa, que ha dexado, y dexa de entrar por los excessivos derechos que cada dia se han ido creciendo, como son quatro reales de puertos, y dos reales y medio de alcavala, y medio de nuevo impuesto, y medio de pesillo, que son siete reales y medio por libra, que vendra a montar catorze, o quinze por ciento ${ }^{27}$.

Ante esta situación se requieren soluciones. En el caso de Juan González, la primera solución que propone para que la seda llegue a Toledo con normalidad relativa es una importante reducción de los impuestos a

\footnotetext{
Ibidem.

lbidem.
} 
los que se ve sometido el producto en la entrada de la ciudad. De este modo, en cifras concretas, el jurado toledano propone que se suprima el nuevo impuesto que supone medio real por parte del ayuntamiento; por parte del rey un real de los cuatro de puertos, un real de los dos y medio de alcabalas y que se pueda vender la seda en martes, día de mercado en Toledo, según privilegio real. Esto supone una reducción de siete reales a dos y medio por lo que la carga tributaria es ostensiblemente menor y la entrada de producto en la ciudad de Toledo es más atractiva. Este tipo de propuestas con el objetivo de paliar un problema determinado, en este caso la bajada de la afluencia de seda en bruto a la ciudad, nos demuestra el complicado mecanismo tributario al que se ven sometidas las mercaderias, desde las materias primas para la actividad manufacturera hasta productos de primera necesidad. La excesiva carga impositiva de la materia prima provoca una subida artificial del precio del producto final que, en el caso de la seda toledana, no puede competir con otros centros donde la carga tributaria es menor, como Madrid o Valencia. En palabras de Juan González de Vatres, la bajada de la carga tributaria sobre la entrada y venta de seda en bruto en Toledo traerá consigo lo siguiente:

...con estos abieros, y baxas de derechos, lo traerán (la seda) manifestado, y sin fraude por los puertos, y entrarà en esta ciudad en la misma forma, y serà mucha la abundancia, y siendolo, suplirà toda esta baxa, y tendrán mucho valor las rentas, como en otras ocasiones se a visto esta consequencia, y no irà a la Villa de Madrid, como es tan gran lugar, y no es cerrado, lo llevan, y los pocos derechos que alli se pagan, se usurpan, donde se han compuesto muchos hombres de negocios que tratan en ello que no solian, sino solo en esta ciudad, y algunas personas van de ella, y lo compran, $y$ traen sin manifestar para bolvello a vender a los Mecaderes fabricantes, $y$ lo entran por donde, y como pueden usurpando, y defraudando todos los derechos a su Magestad, y a V.S. pertenecientes... ${ }^{28}$.

Como vemos, la corrupción es creciente y la entrada de seda de forma ilícita es una práctica que se va generalizando. Este tipo de entradas favorece la especulación, por lo que las fluctuaciones del precio del producto son muy fuertes y afectan sobre todo a los tejidos productivos más débiles, es decir, a los artesanos que por esta razón se ven obligados, en muchos casos, a emigrar o en su defecto a estar desocupados. Estas prácticas fueron muy difíciles de averiguar para los contemporáneos a pesar de tener constancia de su existencia. La solución, aunque parcial, fue la ya citada creación del juez particular de las quince leguas en la cé-

$28 \quad$ A.M.T., Seda, $1500-1650$. 
dula real de 1619. A pesar de la posterior ampliación a veinte leguas de la prohibición de la tenencia de telares en el contorno de Toledo, la inoperancia en el control de entradas y la situación favorable para comerciar con Madrid aumenta las posibilidades de un "contrabando" de seda que provoca una disminución de las rentas municipales y reales gracias a la menor recaudación de impuestos. La solución es clara, bajar la carga tributaria a la seda que entre en la ciudad de forma legal y promocionar el día de mercado franco en Toledo. De este modo

"los que lo traen (la seda) como solian a ella (la ciudad de Toledo), assi a gozar de sus mercados francos, como antes, y despues, lo venderian a precios mas moderados que estos que lo compran para bolvello a vender; los texidos, y demas sedas labradas, y valdrian precios mas acomodados, y no cessara el trato, y comercio desta ciudad, antes ira en gran aumento" ${ }^{29}$.

Además del problema de la especulación que provoca la menor fluidez del comercio terrestre con Toledo, también afecta la llegada de seda ya torcida, lo que hace que un sector integrante del proceso productivo de la seda toledana quede prácticamente inutilizado. Esto supone también el empobrecimiento de una parte dependiente del negocio de la seda, a su vez bastante débil debido a su bajo poder adquisitivo. En este problema es fundamental valorar el desarrollo de esta actividad en las zonas productoras de seda en bruto, como son Valencia y Murcia, que promocionan esta actividad para de este modo exportar la seda ya hilada. Como se indica en el principio del memorial, en la ciudad de Toledo existe una importante ocupación en esta actividad que nos muestra una de las razones del abandono progresivo de la ciudad. La inactividad de los torcedores de seda provoca su traslado a otras zonas que se benefician de su actividad. La ciudad de Valencia, por ejemplo, desarrolla gradualmente una importante industria sedera en detrimento de Toledo. Pero a esto hay que añadir una causa endógena de capital importancia: Toledo no es productora de seda en bruto. Este déficit en la pirámide que constituye la industria sedera en general hace que Toledo sea muy dependiente de las rutas terrestres comerciales con Levante y por tanto, de las comarcas productoras de seda de Murcia y Valencia ${ }^{30}$. La preocupación general de los arbitristas toledanos, y en esto coincide Juan González de Vatres, está en la recuperación de lo perdido en los años de crisis. En el caso de los torcedores de seda,

\footnotetext{
29 Ibidem.

30 Sobre este punto es interesante consultar las obras de James CASEY, op. cit. para el caso de Valencia, y la de Olivares Galván, Pedro, El cultivo y la industria de la seda en Murcia en el siglo xvil. Murcia, Academia Alfonso X el Sabio, 1976, para el caso de Murcia.
} 
Juan González de Vatres propone que se recuperen los tornos que han ido desapareciendo de la ciudad. Esta es su exposición:

Que los tornos de Yladores de seda que avia en esta ciudad, que eran mas de ciento, han faltado, y no ay mas de diez, o doze, porque la seda la solian traer de Valencia, y Murcia por torcer, y aora lo traen torcido, y los tornos que avia en esta ciudad era una cosa muy considerable, y de donde se componia mucho genero de gente que dependia de los fabricantes Yladores, demas de sus casas, y familias, y se puede pedir el remedio conveniente a su Magestad, para que se conserve en esta ciudad esta manifactura de ilar la seda, como oy lo ilan en Valencia, y Murcia, que antes se solia hazer en esta ciudad, por cuya parte le han quitado un gran pedaço de sus fuerças ${ }^{31}$.

Finalmente parece que la conclusión está clara: la importancia que tiene la industria sedera para la ciudad de Toledo. En el plano económico por ser uno de los pilares de la contribución municipal, lo cual demuestra el importante número de habitantes que ocupa tal actividad; en el plano humano, porque supone un contingente poblacional importante dentro de la ciudad. La crisis de esta industria tiene como efectos inmediatos la bajada de las rentas municipales y la despoblación. Las descripciones contenidas en el memorial de Juan González de Vatres Sotomayor son una llamada de atención para poner remedio a la precaria situación en la que se encuentra la industria sedera toledana. La unión de fenómenos tan negativos como la menor afluencia de seda en bruto, materia prima de la industria, un sistema impositivo bastante severo, la creciente especulación y el desmantelamiento progresivo de sectores productivos de la propia industria sedera determinan el binomio que explica la crisis estructural que sufre la ciudad en el primer tercio del siglo XVII: la crisis económica y la despoblación. A pesar de ser un escrito de 1636, las influencias de la literatura económica de los años en torno a 1620 son evidentes, pero también nos prepara para la posterior crisis de 1640. La reflexión de Juan González de Vatres Sotomayor es un ejemplo de la preocupación que tienen importantes sectores del poder sobre la desmantelación económica y humana de la ciudad, preocupación que se irá atenuando con el paso del siglo. Sirva como ejemplo esta última argumentación del jurado toledano:

Tengo entendido que toda esta ciudad, y sus contornos, la mayor dependencia que tiene para su conservacion es de esta fabrica de seda, porque de ella dependen los gastadores de frutos, y demas cosas, porque estando crecido este trato, tiene abundancia de gente, y teniendola, los poderosos tienen caudales, por reditialles sus casas, censos, juros, frutos, y teniendolos crecen

it A.M.T., Seda, 1500-1650. 
sus familias, y sustentan el demas resto de la gente que se ocupan en otros oficios, $y$ a los mismos Mercaderes le compran, $y$ gastan sus fabricas. $Y$ bien se reconoce esto, pues quando avia quatro, o cinco mil telares dentro de Toledo, y cien tornos de Yladores no habia la ruyna que oy tiene, que con lo que le falta se han hecho Otras ciudades poderosas ${ }^{32}$.

\section{ANEXO}

Transcripción íntegra del memorial de Juan González de Vatres Sotomayor (1636):

«loan Gonzalez de Vatres Sotomayor, iurado de esta Imperial Ciudad de Toledo, y Escrivano mayor de rentas Reales de ella, con el zelo de I mayor servicio de V.S. llega a proponer lo que le parece conviene a la conservacion de esta Ciudad, y aumento de la Real hazienda.

Lo primero que el principal comercio, trato y grangeria de esta Ciudad, y donde depende la conservacion de ella es la fabrica de la seda, que se cria en el Reyno de Valencia, y en Murcia, y se trae a esta ciudad en crudo, donde se beneficia por manos de Torcidores, que sustentan mucha gente pobre, a quien la dan a devanar, de donde viene al Mercader que lo comprò, y en su casa con su familia, y otras personas lo adereçan, y entregan a Tintoreros para que lo tiñan de las colores necessarias, los cuales ocupan mucha gente, $y$ de alli lo buelven a entregar al dicho Mercader, que haze muchas suertes, y entrega a mugeres de todos estados para que lo devanen, y pongan en cañones, y açarxas, y de ellas buelve al dicho Mercader, que lo dà a urdir, y hazer telas, y medias a otras personas; $y$ sacado deste urdido, lo entrega a Texedores para que texan estas telas, y listoneria: y estos Texedores tienen sus casas, y familias, que todos se ocupan en disponello, como es en tirar, aviar, hazer canillas, y otras cosas forçosas, y necessarias. $Y$ todo este genero de gente es mucha en esta Ciudad, y en tanto grado, que de las tres partes son mas de las dos, y los que gastan el vestuario, carnes, y frutos de la tierra; y si este trato cessa en esta Ciudad, està acabada totalmente. $Y$ el año que falta el entrar en ella seda en abundancia, se reconoce, porque los Mercaderes, y hombres de negocios que tienen este trato, si les falta de que hazer la fabrica, como tienen tantos gastos, y costas en sus casas, se pierden, y quitan el dar en que entienda toda esta gente, que ordinariamente los mas son pobres, assi hombres, como mugeres, los unos cargados de hijos, otras viudas, y donzellas, que de

32 lbidem. 
afligidos, como les falta el sustento, se van a otras partes a usar de diferentes oficios, y dexan sus casas desamparadas; $y$ por ventura otros dan en vicios temerarios. $Y$ faltando estos gastadores de los mantenimientos, y vestuarios, y no viviendo las casas, los que viven de sus rentas, y frutos de la tierra, estan impossibilitados de labrar sus haziendas, y casas. $Y$ assi es cosa cierta, que por esta falta de haziendas del campo, ni casas de esta ciudad no se labran, fabrican, ni reedifican, antes las dexan hundir, y caer: $y$ de doze años a esta parte en esta ciudad se han caido, y no se avitan el tercio de las casas, y las que han quedado, por la poca gente que ay valen oy muy baxos precios, por grandes que sean. $Y$ este daño, como digo, resulta de no venir a esta ciudad la seda de Valencia, y murcia, porque sus naturales lo dexan de traer por averles cargado, è ido cargando tantos pechos en ella, que les ha obligado a irse a otras partes, donde han dado en hazer las manifacturas que aqui, por averse ido los Texedores, y otros maestros que lo fabrican desta ciudad a ellas, que les han dado la traça, y modo; y en esto es necessario poner remedio, porque sino, se acabarà de hundir esta ciudad, y la Real hazienda va cada dia en diminucion, como lo hemos visto en sus alcavalas, que el año passado de 1628 . valieron mas de 54 . quentos, con que su Magestad pagò, y satisfizo el situado de juros que tiene sobre estas alcavalas, y le sobrò un gran pedaço de dineros, y oy serà harto que valgan 40 . quentos, y cada año han de valer mucho menos ansi ellas, como las atavas de vino, vinagre, y azeite, sisas de carnes, por no aver gastadores: y los Arrendadores que arriendan las rentas, se pierden en ellas, contenellas a mas moderados precios que los que se pierden en ellas, (sic) con tenellas a mas moderados precios que los años passados. $Y$ para que mejor se verifique esta renta de sedas, que es la mas abundante, y de mas consideracion, que valia su alcavala 12. quentos de maravedis, antes mas que menos, baxò el año passado de 1633. a 8 quentos, y 500 . poco mas, o menos, y no por mala administracion, ni porque dexan de pagar lo bastante los contribuyentes en ella, si no por falta de las entradas de la dicha seda; donde se causa, que ha dexado, y dexa de entrar por los excessivos derechos que cada dia se han ido creciendo, como son quatro reales de puertos, y dos reales y medio de alcavala, y medio de nuevo impuesto, y medio de pesillo, que son siete reales y medio por libra, que vendra a montar catorze, o quinze por ciento. $Y$ assi me parece, que quitando V.S. el medio real de nuevo impuesto, se suplique a su Magestad mande se quite un real de los quatro de puertos, y otro real de dos y medio de alcavala: y que el que viniere a vender el martes conforme al previlegio de V.S. venda libremente; $y$ el Mercader fabricante que aquel dia lo comprare, tambien sea libre, pues todo lo suple el dicho previlegio, que su baxa de dichos derechos vendra a ser dos reales y medio por libra, de los siete reales y medio que paga, sin 
Corredor, que tambien lo mandarà V.S. minorar, y que por esta parte no se les haga vexaciones a los forasteros. $Y$ con estos abieros, $y$ baxas de derechos, lo traeran manifestado, y sin fraude por los puertos, y entrarà en esta ciudad en la misma forma, y serà mucha la abundancia, y siendolo, suplirà toda esta baxa, $y$ tendran mucho valor las rentas, como en otras ocasiones se a visto esta consequencia, y no irà a la Villa de Madrid, como es tan gran lugar, y no es cerrado, lo llevan, y los pocos derechos que alli se pagan, se usurpan, donde se han compuesto muchos hombres de negocios que tratan en ello que no solian, sino solo en esta ciudad, y alguanas personas van de ella, y lo compran, y traen sin manifestar para bolvello a vender a los Mercaderes fabricantes, y lo entran por donde, y como pueden, usurpando, y defraudando todos los derechos de su Magestad, y a V.S. pertenecientes; y esto es caso imposible de averiguar, sino es que tal vez se coxa la misma seda. Y es conocido el gran daño del comercio, trato, y negocio de la seda en esta ciudad, porque si los que los traen como solian a ella, assi a gozar de sus mercados francos, como antes, y despues, 10 vendieran a precios mas moderados que estos que lo compran para bolvello a vender; los texidos, y demas sedas labradas, y valdrian precios mas acomodados, y no cessaria el trato, y comercio desta ciudad, antes ira en gran aumento; porque esto no sirve mas que de hazerse algunos (que seran pocos) poderosos, y los demas pobres, y sin caudal. $Y$ es cierto que no avia fraudes en las entradas, pagando tan solamente de todos los derechos cinco reales por libra: porque el que quiere que le traigan su seda bolada (que llaman) le cuesta de cada libra el que se lo trae hasta los limites de esta ciudad dos reales y medio, y el que lo entra en ella, por lo menos le lleva un real por libra, y despues de entrado, si lo han de vender encubiertamente, pierde por lo menos otro real por libra, que vienen a ser quatro reales y medio demas del riesgo a que se ponen, y respeto desto se manifiestara todo: que claro estâ que no teniendo grangeria ninguna, nadie querra aventurar su hazienda, y al comercio, de tres partes de las dos. Porque desde el año 1628. a esta parte he visto, que aviendose aliviado a los mercaderes de seda en sus conciertos, y pechas quatro quentos de maravedis poco mas, 0 menos, de un año a otro de lo que pagavan, por estar cargados, y hechar buen passage a la seda que entrô de fuera pagando menos derechos, suplio esta baxa de quatro quentos, la grande entrada de seda, y manifestacion que hubo de ella, y tuvo esta renta su valor de doze quentos, y mas aviendose baxado de ella costas, y gastos, y despues se alteraron los conciertos, y pechas, pareciendo se hazia el servicio de su Magestad, y se apretò la entrada de la seda, que estava suavizada, y baxô de los doze quentos un pedaço, y se quitaron por este crecimiento que se hizo a las pechas muchos negocios, con que este gremio de la seda, y renta ha venido en tan gran di- 
minucion como se ve, que de tres partes a faltado la una, y lo mismo ha sido la gente, y comercio de la Ciudad, en tan breve tiempo, y si no se pone remedio por V.S. va tan apriessa, que quando se quiera remediar no ha de ser posible.

Que V.S. pidiesse a su Magestad, se pudiesse a execucion, como està mandado assi por concessiones de millones que V.S. ha hecho, como en otros servicios, que en la Corte donde està su Real persona, no asistiessen fabricantes de texidos, sino que cada uno se fuesse a su tierra a fabricallos, que con esto le tocarà a esta ciudad la mayor parte, porque lo mas que fabrican es texidos de sedas, que todos solian labrarse en esta ciudad. $Y$ viniendo estos fabricadores, vendrian tambien con ellos los Mercaderes fabricantes, porque el Mercader fabricante salio de su natural para ir a la Corte, donde hallò de que hazer la fabrica con mas comodidad y privandole de que no fabrique en ella, bolverà a su natural, donde hallando las conveniencias arriba referidas, y que tenia en la Corte, se conservarà en el, y serâ fuerça que los texidos que le compravan los Mercaderes vareantes en la Corte para su despacho, se les vengan a comprar a esta ciudad, como lo han acostumbrado, y acostumbran; y procurando hazerles buen passage a ellos, y los forasteros que vinieren a comprar los texidos. $Y$ los que traxeren seda para ellos creceran los caudales, y haziendas de los que vinieren, y de los que oy ay, y no empobrezeran, como lo van haziendo, y dexando los tratos, y negocios por la poca grangeria que hallan, y mal despacho que tienen, por fabricarse en otras partes los texidos que tan solamente en esta ciudad se fabricavan; y los que dexan, dan en contentarse con un poco de renta, y minorar sus familias, y el que lo dexa destruye muchos pobres de todos estados, que vivian con la manifactura que hazen del Mercader fabricante. $Y$ los que no lo dexan, es, porque viven con el credito, y si este les falta, quedaran pobres, y de fuerça no se atreven a ajustar su cuenta, porque no se vea, hasta que el tiempo se la descubre, como se ha visto.

Que los tornos de Yladores de seda que avia en esta ciudad, que eran mas de ciento, han faltado, y no ay mas de diez, o doze, porque la seda la solian traer de Valencia, y Murcia por torcer, y aora lo traen torcido y los tornos que avia en esta ciudad los han puesto alla, y esto en esta ciudad era una cosa muy considerable, y de donde se componia mucho genero de gente que dependia de los fabricantes Yladores, demas de sus casas, y familias, y se puede pedir el remedio conveniente a su Magestad, para que se conserve en esta ciudad esta manifactura de ilar la seda, como oy lo ilan Valencia, y Murcia, que antes se solia hazer en esta ciudad, por cuya parte le han quitado un gran pedaço de sus fuerças.

Tengo entendido que toda esta ciudad, y sus contornos, la mayor dependencia que tiene para su conservacion es de esta fabrica de seda, 
porque de ella dependen los gastadores de frutos, y demas cosas, porque estando crecido este trato, tiene abundancia de gente, y teniendola, los poderosos tienen caudales, por reditialles sus casas, censos, juros, frutos, y teniendolos crecen sus familias, y sustentan el demas resto de la gente que se ocupan en otros oficios, y a los mismos Mercaderes les compran, y gastan sus fabricas. $Y$ bien se reconoce esto, pues quando avia quatro, o cinco mil telares dentro de Toledo, y cien tornos de Yladores no habia la ruyna que oy tiene, que con lo que le falta se han hecho otras ciudades poderosas. Y a V.S. como pader, y amparo de esta Republica le toca su remedio, y pues en otras ocasiones que a V.S. se le han ofrecido, ha suplicado a su Magestad, no tan solamente por lo que le toca, sino por todo el Reyno ha sido oydo de manera, que su Magestad fue servido, y remediado su Reyno.

Suplico a V.S. en la forma que puedo, se sirva de nombrar sus Cavalleros Comissarios, para que representen a su Magestad el estado que esta ciudad tiene, y ponga remedio a su ruina, con lo mas que a V.S. le ocurriere con sus grandes ingenios, a quien suplico perdone mi atrevimiento, y como hijo reciba mi voluntad. 